\title{
Concepto, aplicación y modelo de prospectiva estratégica en la administración de las organizaciones
}

Carlos William Mera Rodríguez'

"El futuro no se predice, sino se construye".

Maurice Blondel

\section{Resumen}

En un mundo globalizado y complejo, como el de hoy, se hace necesario que los administradores conozcan y utilicen una herramienta vital para construir el futuro de las organizaciones: la prospectiva estratégica. En este documento se busca dar a conocer su origen, las diferentes conceptualizaciones de la prospectiva, y sus aplicaciones en los diferentes ámbitos; por último, se presenta uno de los modelos y metodologías utilizados actualmente en el desarrollo de ejercicios prospectivos.

Palabras clave: Prospectiva, estrategia, futuro, modelo prospectivo, administración.

\section{Abstract}

In a globalized and complex, like today, it is necessary that managers know and use a vital tool for building the future of the organization: strategic foresight. This paper seeks to publicize their origin, the different conceptualizations of foresight, and their applications in various areas, and finally, presents one of the models and methodologies currently used in the development of prospective exercises.

Keywords: Prospective, strategy, future prospective model, administration.

Recibido: 16 de abril de 2012

Aceptado: 24 de julio de 2012

\section{Los orígenes de la prospectiva}

La escuela francesa de la prospectiva nace a finales de los años cincuenta del siglo XX, gracias a las construcciones teóricas de los filósofos y pensadores Gastón Berger y Beltrand de Jouvenel, la cual se fundamenta en la identificación de futuros posibles, o "futuribles," para escoger el más conveniente y construirlo desde el presente.

Pero es durante los años ochenta del siglo XX cuando el profesor Michel Godet, comienza a visibilizar la prospectiva a través de ejercicios prospectivos académicos y con múltiples empresas, y durante los años noventa, crea el LIPSOR (Laboratorio de Investigación Prospectiva y Estratégica de las Organizaciones), en el CNAM (Conservatorio Nacional de Artes y Oficios), la cual tenía por objetivo formar en el campo de la prospectiva a profesionales que apoyaran la construcción del futuro a través de la prospectiva de empresas privadas y públicas.
América Latina no ha sido ajena a esta corriente. Gracias a la labor de Organización de las Naciones Unidas para el Desarrollo Industrial (ONUDI), desde 1998, en Latinoamérica y el Caribe se vienen implementando los respectivos Programas Nacionales de Prospectiva en Brasil, Argentina, Venezuela, Colombia, Uruguay, Ecuador, Chile y México; todos estos países vienen ejecutando con gran éxito sus programas nacionales. Brasil se destaca por haber logrado llevar los resultados de sus estudios de prospectiva a los niveles de decisión política y al logro de un consenso Estado-academia-sector privado-sindicatos. Las cadenas productivas priorizadas hasta ahora en el Brasil son: construcción civil, textiles y confecciones, madera y muebles, y plásticos. El horizonte de planeamiento empleado es 2050.

El Perú ingresó tarde a esta corriente de planeamiento, pero las instituciones que conformaban el comité organizador de PROSPECTA PERÚ 2003 se empeñaron en recuperar el tiempo perdido. Durante la última década, 
formaron una masa crítica de más de 500 profesionales capacitados en el empleo de las principales metodologías usadas en la formulación de los estudios de prospectiva, la experiencia exitosa más representativa de la actualidad es el ejercicio prospectivo PLAN PERÚ 2040, el cual se desarrolla con el apoyo del gobierno nacional y departamental. Esta experiencia es un ejemplo para la región, y ha sido presentada en varios países como un caso exitoso para seguir.

Pero la prospectiva no ha sido empleada solo por los gobiernos. Es en el campo empresarial donde ha tenido un impacto significativo a lo largo de las tres últimas décadas. La primera empresa multinacional que empleó una de las metodologías de prospectiva, el planeamiento por escenarios, fue la petrolera Royal Shell, en 1968. Antes de esa fecha, la Shell solo aplicaba métodos de pronóstico, lo cual les impedía identificar e interpretar eventos cruciales para su industria, como el ocurrido en octubre de 1973 en el Medio Oriente, y que luego fue conocido como "la crisis del petróleo de 1973". Al aplicar las técnicas de prospectiva, la Shell pudo vislumbrar dicho escenario, tan poco deseable, y plantear planes contingentes que fueron empleados en su debido momento. Según Ortega (1998), "La prospectiva ha tenido un impacto significativo en el campo empresarial en las últimas tres décadas. La primera empresa multinacional que empleó una de las metodologías prospectivas, el pensamiento por escenarios, fue la petrolera Royal Shell en 1968."

En la actualidad, la mayoría de las grandes empresas internacionales, desde las automotrices hasta las líderes de las microelectrónicas y las telecomunicaciones, así como bancos de inversión y trading companies, emplean la prospectiva para el planeamiento de mediano y largo plazo de sus operaciones.

En Colombia la aplicación de la prospectiva se ha venido desarrollando de forma dinámica a lo largo de la última década, gracias al arduo trabajo de los prospectivistas Francisco José Mojica y Javier Medina, y durante los últimos años, con mayor impulso, por iniciativa de jóvenes prospectivistas discípulos de estos maestros. En la actualidad se vienen desarrollando múltiples ejercicios prospectivos en el ámbito de los gobiernos central, departamental y municipal; de igual forma, se realizan estudios prospectivos en organizaciones, universidades y en los diferentes sectores económicos del país.

Otros factores de éxito han sido la visibilidad y la divulgación de estos ejercicios prospectivos a través de diferentes congresos, seminarios y foros de prospectiva, en gran parte desarrollados por las universidades líderes en el tema de prospectiva, tales como: la Universidad Externado de Colombia, La Universidad del Valle y La Universidad Nacional Abierta y a Distancia; esta última organiza cada año un congreso internacional donde se presentan los resultados de investigaciones, estudios, ejercicios y experiencias exitosas de prospectiva en el mundo entero.

\section{¿Qué es la prospectiva?}

Berger (1964) uno de los fundadores de la disciplina, la define como "la ciencia que estudia el futuro para comprenderlo y poder influir en él." La prospectiva parte del concepto según el cual el futuro aún no existe, y "se puede concebir como una realizar múltiple" Jouvenel (1993), y que "depende solamente de la acción del hombre" Godet (1990). Por tal razón, el hombre puede construir el mejor futuro posible, y para ello debe tomar las decisiones correctas en el momento apropiado.

Jouvenel (1993) plantea que existen dos formas de ver el futuro: la primera, como una realidad única, propia de los oráculos, los profetas y los adivinos. La segunda, como una realidad múltiple. Estos son los futuros posibles que denominó futuribles.

Balbi (2008) define la prospectiva como la disciplina que ha logrado convertirse en la herramienta clave de esa construcción del futuro deseado y posible; en particular, sus últimos desarrollos, que arriban a la llamada, prospectiva estratégica, que constituyen el basamento fundamental del liderazgo y del management moderno.

Gil (2005) concibe la prospectiva de dos formas: como una "disciplina intelectual" o como una "indisciplina intelectual" que toma la forma de una "reflexión para iluminar la acción del presente con la luz de los futuros posibles,' en palabras del francés Godet (1993). Esta disciplina propone analizar las posibles evoluciones de una organización o territorio -o parte de él- en un horizonte de tiempo determinado, teniendo en cuenta las interacciones que estos tiene con sus entornos endógenos y exógenos, para de, esta forma, elegir su mejor alternativa de futuro posible, a través de un ejercicio colectivo donde convergen todas las voluntades de los actores relacionados con dicha organización o territorio, como una herramienta de construcción social del futuro que motiva y permite la generación de una cultura "anticipatoria"

Ben Martin (1995) describe la prospectiva como "El proceso de investigación que requiere mirar sistemáticamente el futuro de largo plazo en ciencia, tecnología, economía y sociedad, con el objetivo de identificar las áreas de investigación estratégicas y las tecnologías genéricas emergentes que generarán los mayores beneficios económicos y sociales."

En su libro "La sociología de la previsión", Decouflé (1973) comenta que "hablar de futuro" puede tomar seis formas del discurso teórico-práctico, y las cuales se resumen en la siguiente tabla: 
Tabla 1: discurso teórico-práctico

\begin{tabular}{|l|l|}
\hline \multicolumn{1}{|c|}{ Discurso teórico-práctico } & \multicolumn{1}{c|}{ Designación aceptada } \\
\hline Adivinar & Adivinación \\
\hline Predecir & Profecía \\
\hline Anticipar & Futurología \\
\hline Construir el futuro & Prospectiva \\
\hline Imaginar & Ciencia-Ficción \\
\hline Soñar & Utopía \\
\hline
\end{tabular}

\section{¿Qué es la prospectiva estratégica?}

Si bien es cierto que la prospectiva permite ver las alternativas de futuro, también es importante plantearse preguntas del tipo: ¿qué vamos a hacer?, y ¿Cómo lo vamos a hacer? Esto se logra mediante la articulación de la prospectiva y la estrategia, Godet (2007).La estrategia nos dice como construir el futuro que más conviene. La una sin la otra no tendría sentido. La prospectiva consiste en la exploración de los futuros posibles, es decir, de lo que puede acontecer. La estrategia consiste en lo que puede hacerse. Ambas disciplinas son indispensables en el mundo actual y debemos familiarizarnos con ellas, si queremos estar en la frontera de la competitividad.

Por estas razones, es que en Colombia, y específicamente en los ámbitos académico y universitario, se ha tomado como modelo la prospectiva estratégica y se la aplica en la gran mayoría de ejercicios prospectivos realizados en el país, con algunas excepciones que manejan otros modelos igualmente eficaces.

\section{¿Cuáles son los objetivos de la prospectiva estratégica?}

- Construir escenarios alternativos de futuro.

- Hacer explícitos escenarios alternativos de futuros posibles.

- Promover información relevante bajo un enfoque de largo plazo.

- Establecer valores y reglas de decisión para alcanzar el futuro deseado.

- Proporcionar impulsos para la acción.

\section{Características de la prospectiva estratégica}

- Trabaja con una perspectiva sistémica, holística y compleja.

- Es interdisciplinaria.

- Se requieren creatividad e imaginación para crear algo nuevo y valioso.
- Es participativa; busca alcanzar consensos, involucrar a los actores, buscar compromisos.

- Es un proceso sistemático y continuado en el tiempo.

- Hay preminencia del proceso sobre el producto.

- Asume que todas las variables están relacionadas de forma dinámica entre ellas, y considera que el futuro se puede moldear desde el presente.

- Integra parámetros cualitativos, tales como el comportamiento de los actores.

- Su finalidad es constructora, está orientada a la acción.

\section{¿Por qué la importancia de la prospectiva?}

Los cambios que se están produciendo en los ámbitos social, económico, político, cultural, tecnológico y ambiental son tan importantes, tan profundos ( $\mathrm{y}$ algunos de ellos, a un ritmo tal), que ya han comenzado a sentirse los efectos en las instituciones/organizaciones y las empresas. En este contexto de incertidumbre no son suficientes los análisis tradicionales que se alimentan desde y con datos exclusivos del pasado, sino que se hace imprescindible disponer, además, de nuevas herramientas que permitan tener visiones de los posibles escenarios en cada uno de los mencionados ámbitos, y solo así podrán adoptarse las estrategias correctas para lograr el escenario deseado.

En el presente artículo se centra la discusión en el ámbito de la educación, y, específicamente, en el tema de la administración; por tal motivo, en este documento se presentará la importancia de la prospectiva estratégica, la metodología utilizada para desarrollar ejercicios prospectivos en las organizaciones.

Para empezar. la prospectiva actualmente se utiliza para explorar las posibles o probables evoluciones futuras de las organizaciones, las empresas, los sectores comerciales, los sectores productivos, las instituciones públicas o las instituciones privadas, como una herramienta que a todos ellos les permite visualizar a futuro problemáticas de índole social, económica, política, ambiental, tecnológica y cultural, etc., a mediano y lar- 
go plazo, con el apoyo y el soporte de los modelos de la prospectiva estratégica, su metodología, sus herramientas y sus técnicas.

\section{Aplicaciones de la prospectiva estratégica}

Balbi (2003) presidente de la Red de Escenarios y Estrategias para América Latina, plantea las siguientes aplicaciones directas de la prospectiva estratégica:

\section{Prospectiva territorial}

Se desarrolla en la organización de un territorio, sus recursos y su gente, para el desarrollo sustentable, la alta calidad de vida, el cuidado del ambiente y el logro de la seguridad humana.

\section{Prospectiva urbana}

Se utiliza en las organizaciones de áreas urbanas, sus recursos, su gente y sus áreas satélites, para el desarrollo sustentable, la alta calidad de vida, el cuidado del ambiente y el logro de la seguridad humana.

\section{Prospectiva de seguridad}

Se utiliza en el análisis de los riesgos y de las oportunidades en seguridad física, ambiental, industrial y del trabajo, frente a situaciones normales, excepcionales o de crisis. En Colombia se ha desarrollado ampliamente este tema en las Fuerzas Miliares y en la Policía Nacional.

\section{Prospectiva organizacional}

Es la que se encarga dl estudio de futuro de las diferentes organizaciones y pretende darle respuesta a I preguntas como ¿Cuál deberá ser la organización, misión, tareas y competencias de mi organización en el año X?

\section{Prospectiva Competitiva}

Se aplica a la organización y la puesta en marcha de sistemas integrados de producción, servicios, exportación, negocios, (clúster, cadenas, consorcios), etc.

\section{Prospectiva científica, tecnológica y de in- novación}

Diseños de políticas, estrategias y programas para lograr la Innovación y Desarrollo (I\&D) en Ciencias y Tecnología (C\&T) y las innovaciones necesarias para el desarrollo sustentable de regiones, ciudades y sectores.

Mera (2010) adiciona dos aplicaciones directas de la prospectiva estratégica al planteamiento del profesor Eduardo Balbi:

\section{Prospectiva Social}

Se aplica en la construcción de escenarios alternativos para la solución de problemáticas sociales como la exclusión social, la inequidad, la solidaridad, el emprendimiento social solidario, y la economía social y la residencia.

\section{Prospectiva comunitaria}

Promueve la construcción comunitaria del futuro en los ámbitos sociales, comunitarios y organizacionales, con el fin de fortalecer la toma de decisiones, disminuir el costo social y mejorar la calidad humana en el planeta.

\section{Metodología de la prospectiva estratégica}

El modelo "avanzado" de prospectiva estratégica de la "escuela voluntarista" es una adaptación del profesor Francisco José Mojica (2008) ${ }^{2}$ a las condiciones propias del entorno colombiano y latinoamericano.

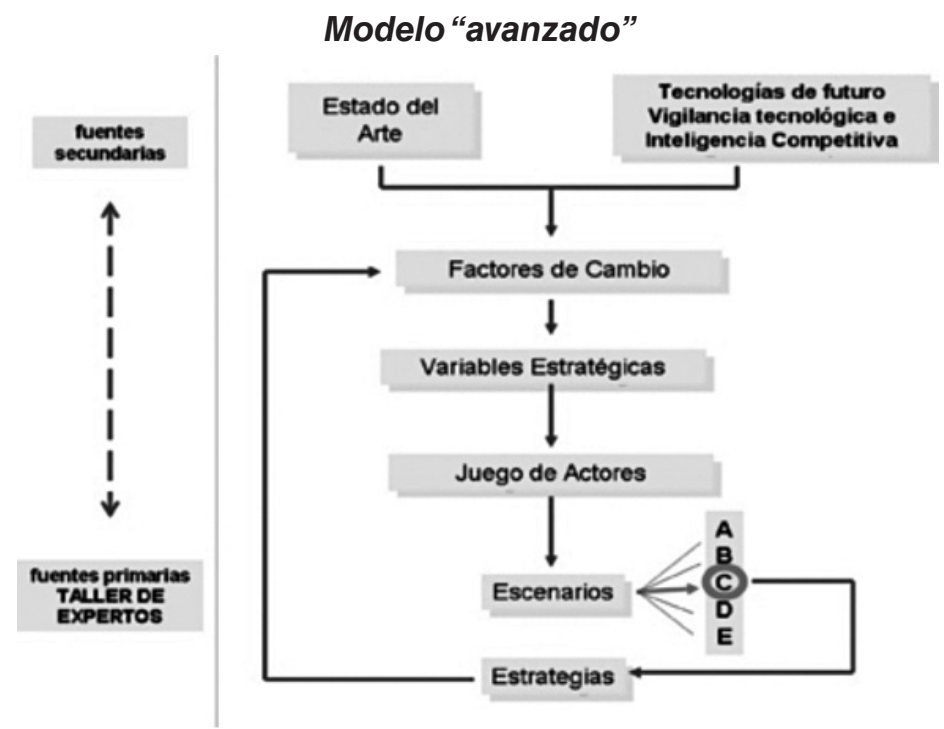

Gráfico 1. Modelo de mayor complejidad de la prospectiva estratégica

Fuente: Francisco José Mojica 


\section{Revisión de fuentes secundarias}

Se realiza un estado del arte, lo cual no es otra cosa que ir tras las huellas del objeto de estudio. En esta fase del trabajo, la situación actual del programa, cabe preguntarse cómo es hoy el programa; también, cómo era en el pasado y qué estudios se han realizado sobre el futuro de la administración. De igual manera, se lleva a cabo un estudio de vigilancia tecnológica e inteligencia competitiva, que permita identificar las tendencias mundiales en lo educativo; y en lo tecnológico, y así como las mejores prácticas. Para el desarrollo de estas etapas se utilizaron bases de datos, buscadores de datos especializados, tanques de pensamiento, centros de investigación, centros de gestión de conocimiento especializado y observatorios.

\section{Información de las fuentes primarias}

En esta etapa se diseña y se aplica una encuesta Delphi a expertos, considerados conocedores del tema que se va a desarrollar.

\section{Identificación de factores de cambio}

Los factores de cambio son las características de la organización que se expresan en fenómenos económicos, sociales, culturales, tecnológicos, políticos, ambientales o educativos, entre otros. En esta etapa se pretende, con la ayuda de una encuesta Delphi y la de los expertos participantes, identificar cuáles son los factores de cambio que incidirán en el futuro de la administración. Para el presente estudio se seleccionó como herramienta de apoyo la Matriz del Cambio de Michel Godet ${ }^{3}$, la cual permite identificar los cambios temidos, presentidos y anhelados en el futuro.

\section{Selección de las variables estratégicas}

Es importante tener en cuenta que no todos los factores encontrados durante la etapa anterior son importantes: lo son solo algunos, a las que se llamará variables estratégicas o variables clave. Para la selección de dichas variables se utilizará el "análisis estructural", que Michel Godet diseño con el nombre de "MicMac," y es un aporte a la "Caja de herramientas", herramienta muy sencilla como el análisis estructural, denominado técnicamente Método Micmac.

\section{Juego de actores}

Esta etapa tiene por objetivo estudiar el comportamiento de actores sociales que tienen relación con las variables estratégicas seleccionadas. En ella se determinan los retos, los conflictos, las posibles jugadas o las estrategias que se deben diseñar para lograr el escenario apuesta.

\section{Construcción de escenarios}

Para la construcción de los escenarios se empleará una técnica creada por Shwartz (1993) conocida como los "ejes de Schwartz", con la cual se van a plantear los escenarios posibles, tendenciales, exploratorios y apuesta. En esta etapa no solo se diseñaran los escenarios, sino que se harán un análisis y unas explicación de cada uno de los escenarios, lo cual permitirá la elección de un "escenario apuesta".

\section{Diseño de estrategias}

La última etapa del modelo es la definición de estrategias, la cual se desarrollará a través de un plan prospectivo y estratégico, compuesto por los objetivos estratégicos, las metas, las actividades, las acciones, las instituciones que intervienen, los responsables y los planes de seguimiento.

Es importante en esta etapa del modelo definir con claridad los términos estrategia, planeamiento estratégico y planeamiento prospectivo estratégico.

Fred (2003) define estrategia como los medios para lograr los objetivos. Por otra parte, Drucker (1994) fue uno de los primeros en mencionar el término estrategia en la administración; para él, estrategia de la organización era la respuesta a dos preguntas: ¿Qué es nuestro negocio? y ¿Qué debería ser? Y Mintzberg (1997) plantea que la estrategia es el patrón o plan que integra las principales metas y políticas de una organización, y, a la vez, establece la consecuencia coherente de las acciones por realizar.

Miklos (2001), por su parte, define la planeación estratégica como el esfuerzo sistemático, y más o menos formal, de una institución u organización para establecer sus propósitos, sus objetivos, sus políticas y sus estrategias básicas, con el fin de desarrollar planes detallados que pongan en práctica las políticas y las estrategias que la lleven a lograr sus objetivos y sus propósitos fundamentales.

Godet (1990) define el "Planeamiento Estratégico Prospectivo como un nuevo enfoque de Planeamiento que consiste en guiar a todos los Planeamientos Estratégicos de la organización hacia un futuro, creando escenarios futuros que desea alcanzar la organización, tomando medidas correctivas para un presente más deseado y para alcanzar aquel escenario posible, deseable y probable". 


\section{Referencias bibliográficas}

Balbi, E. (2003). La técnica de análisis de causas y efectos, y remplazo del comportamiento de indicadores (CEYR). En Balbi, E. (editor). Metodología de investigación de futuros. Metodología prospectiva (CD). Buenos Aires: Editor Eduardo Raúl Balbi.

Balbi, E. (2008). Metodología de la investigación de futuros: CELGYP y The millennium project. Buenos Aires: Editor Eduardo Raúl Balbi.

Berger, G. (1967). Gastón. Etapes de la prospective. Paris: PUF.

Berger, G. (1957). Sciences humaines et previsión. Revue des Deux Mondes 1. París, Francia

Decouflé, A. (1973). La prospectiva. Paris: Editorial Oikos-Tau.

Drucker, P. (1994). The theory of business. Cambridge: Harvard Business Review.

Fred, D. (2003). Conceptos de administración estratégica. México: Pearson-Prentice Hall.

Gil, B. (2005) "Inteligencia científica, tecnológica y regional." Manual del participante del Diplomado regional en gestión del conocimiento. Bogotá: Unisabana-Colciencias.

Godet, M. (2000) "L'art et la method" en Godet, M. "Manuel de Prospective Estratégique" (tome 2, p. 227). Paris: Dunod.

Godet, M. (2000). La caja de herramientas de la prospectiva estratégica, recuperado de www.prospektiker. es/documentos/caja2000.pdf.

Godet, M, (1991). Problemas y métodos de prospectiva: caja de herramientas. Donostia, San Sebastián (País Vasco): Prospektiker-Futuribles, UNESCO.

Jouvenel, H.(1993). Sur la méthode prospective: un bref guide méthodologique, Futuribles, 179.

Lipsor, (2005). Analyser les stratégies d'acteurs, recuperado de http://www.3ie.org/lipsor/mactor.htm

Lipsor (2005). MICMAC-Se poser les bonnes questions et identifier les variables clés, recup e rado de http://www.3ie.org/lipsor/micmac.htm.

Lipsor (2005). SMIC-PROB-EXPERT, Impacts croisés probabilistes, recuperado de http://www.3ie.org/lipsor/ mactor.htm.
Mera, C. (2009). La prospectiva estratégica herramienta para construir la esencia social Comunitaria en las regiones al 2015. Foro Nacional de la Esencial Social Comunitaria. Bogotá: UNAD.

Mera, C. (2010). Curso de profundización en prospectiva estratégica por escenarios empresariales. Universidad Nacional Abierta y a Distancia. Bogotá: UNAD.

Methodes de Prospective, recuperado de www.laprospective.fr/methodes-de-prospective.html

Miklos, T., et al. (Coord). (2002). Las decisiones politicas, de la planeación a la acción. México: Siglo veintiuno editores.

Mintzberg, H., Quinn, J. \& Voyer, J. (1997). El proceso estratégico: Concepto, contextos y casos. México: Prentice Hall-Pearson Educación.

Mojica, F. J. (2006). Concepto y aplicación de la prospectiva estratégica. Revista Med, julio, 122-131.

Mojica, J. (2005). La construcción de futuro. Bogotá: Editorial Universidad Externado de Colombia/Convenio Andrés Bello.

Mojica, J. (2008). Dos modelos de la escuela voluntarista de prospectiva estratégica. Bogotá: Universidad Externado de Colombia.

Reseñas http://books.google.com/books/about/Conceptos_de_Administraci\%C3\%b3n_Estrat\%C3\%a9gic. html?HI=es\&id=l7ethj2twfoc

Schwartz, P. (1991). The art of the long view. New York: Currency Doubleday.

Schwartz, P. (1993). La planification stratégique par scénarios. Revista Futuribles, 176.

\section{Notas}

2 Francisco José Mojica es doctor en ciencias humanas de la Universidad de París V "René Descartes". Estudió prospectiva de la mano del profesor Michel Godet, en el Lipsor de París. Actualmente dirige el Doctorado en Ciencias de la Administración, así como el "Centro Pensamiento Estratégico y Prospectiva," de la Universidad Externado de Colombia, en Bogotá.

${ }^{3}$ La "matriz del cambio" de la caja de herramientas de Michel Godet.

${ }^{4}$ Con el nombre de "Caja de Herramientas", Michel Godet tituló una de sus obras, que contiene las diferentes técnicas prospectivas producidas por él. Esta obra esa en formato electrónico, y se la puede descargar de la página web del autor: www.laprospective.com 